PROCEEDINGS OF THE

AMERICAN MATHEMATICAL SOCIETY

Volume 125, Number 11, November 1997, Pages 3229-3234

S 0002-9939(97)04221-4

\title{
CHARACTERIZATION FOR BEURLING-BJÖRCK SPACE AND SCHWARTZ SPACE
}

\author{
SOON-YEONG CHUNG, DOHAN KIM, AND SUNGJIN LEE
}

(Communicated by J. Marshall Ash)

\begin{abstract}
We give an elementary proof of the equivalence of the original definition of Schwartz and our characterization for the Schwartz space $\mathcal{S}$. The new proof is based on the Landau inequality concerning the estimates of derivatives. Applying the same method, as an application, we give a better symmetric characterization of the Beurling-Björck space of test functions for tempered ultradistributions with respect to Fourier transform without conditions on derivatives.
\end{abstract}

\section{INTRODUCTION}

In [3], [4], [5] new characterizations for the Schwartz space $\mathcal{S}$, the Sato space $\mathcal{F}$ of test functions for the Fourier hyperfunctions, and the Gelfand-Shilov spaces of type $\mathrm{S}$ and type $\mathrm{W}$ have been given in terms of Fourier transformation as follows.

I. [3] For the Schwartz space $\mathcal{S}$ the following statements are equivalent.

(1) $\varphi \in \mathcal{S}$.

(2) $\sup _{x}\left|x^{\alpha} \varphi(x)\right|<\infty, \quad \sup _{x}\left|\partial^{\beta} \varphi(x)\right|<\infty$

for all multi-indices $\alpha$ and $\beta$.

(3) $\sup _{x}\left|x^{\alpha} \varphi(x)\right|<\infty, \quad \sup _{\xi}\left|\xi^{\beta} \hat{\varphi}(\xi)\right|<\infty$,

for every $\alpha$ and $\beta$, where $\hat{\varphi}$ is the Fourier transform of $\varphi$.

II. [4] For the Sato space $\mathcal{F}$ the following statements are equivalent.

(1) $\varphi \in \mathcal{F}$.

(2) There exist positive constants $h, k>0$ such that

$$
\sup _{x}|\varphi(x)| \exp k|x|<\infty, \quad \sup _{\xi}|\hat{\varphi}(\xi)| \exp h|\xi|<\infty .
$$

III. [5] For the Gelfand-Shilov space $S_{\alpha}^{\beta}$ of type $\mathrm{S}$ the following statements are equivalent.

(1) $\varphi \in S_{\alpha}^{\beta}$.

(2) $\sup _{x}|\varphi(x)| \exp k|x|^{1 / \alpha}<\infty, \quad \sup _{\xi}|\hat{\varphi}(\xi)| \exp h|\xi|^{1 / \beta}<\infty$

for some $h, k>0$.

Received by the editors December 11, 1995.

1991 Mathematics Subject Classification. Primary 46F05, 46F12, 42B10.

Key words and phrases. Fourier transform, Schwartz space, Beurling-Björck space, tempered, ultradistributions.

This work was partially supported by GARC-KOSEF and BSRI.

(C) 1997 American Mathematical Society 
IV. [5] For the Gelfand-Shilov space $W_{M}^{\Omega}$ of type $\mathrm{W}$ the following statements are equivalent.

(1) $\varphi \in W_{M}^{\Omega}$.

(2) $\sup _{x}|\varphi(x)| \exp M(a|x|)<\infty, \quad \sup _{\xi}|\hat{\varphi}(\xi)| \exp \Omega^{*}(b|\xi|)<\infty$

for some $a, b>0$, where $\Omega^{*}$ is the Young conjugate of $\Omega$.

Here we use the multi-index notation: $x^{\alpha}=x_{1}^{\alpha_{1}} \cdots x_{n}^{\alpha_{n}}, \partial^{\alpha}=\partial_{1}^{\alpha_{1}} \cdots \partial_{n}^{\alpha_{n}}$ for $\alpha \in \mathbb{N}_{0}^{n}$, where $\mathbb{N}_{0}$ is the set of nonnegative integers and $\partial_{j}=\partial / \partial x_{j}$.

In this paper we first give an elementary proof of result I, in other words, the equivalence of the original definition of Schwartz in [12] and our characterization in [3] for the Schwartz space $\mathcal{S}$ in Section 2. A new proof is based on the Landau inequality concerning the estimates of derivatives. Also, we can easily apply the above method to obtain better definitions for the spaces $H\left(\mathbb{R}^{n}\right), S_{1}$ and $\mathcal{S}_{E}$, introduced by Hasumi [10], Gelfand-Shilov [9] and Chung-Kim [6], respectively.

In Section 3, applying the same method, as an application, we give a better characterization of the Beurling-Björck space $\mathcal{S}_{\omega}$ of test functions for tempered ultradistributions in [1] by removing conditions on the derivatives in Definition 3.1 in a similar fashion as in the above results I-IV. In other words, we show that the assumption for the decay of derivatives is redundant. Finally, we show that the Silva space $\mathcal{G}$ of test functions for the Fourier ultrahyperfunctions is nothing but a special case of the Beurling-Björck space when $\omega(x)=|x|$.

The functional analysis and Fourier analysis in the dual space $\mathcal{S}_{\omega}^{\prime}$ of tempered ultradistributions have been extensively studied in [11].

\section{Elementary proof for characterization of the Schwartz space}

L. Schwartz introduced the Schwartz space $\mathcal{S}$ or $\mathcal{S}\left(\mathbb{R}^{n}\right)$ of all infinitely differentiable functions $\varphi$ satisfying

$$
\sup _{x}\left|x^{\alpha} \partial^{\beta} \varphi(x)\right|<\infty
$$

for all multi-indices $\alpha$ and $\beta$ in his famous treatise [12].

If $\varphi \in \mathcal{S}$, then it easily follows from (2.1) that

$$
\sup _{x}\left|x^{\alpha} \varphi(x)\right|<\infty, \quad \sup _{x}\left|\partial^{\beta} \varphi(x)\right|<\infty
$$

for all multi-indices $\alpha$ and $\beta$. In [3] we show that the converse also holds true. In other words, the condition (2.2) characterizes the Schwartz space $\mathcal{S}$ as follows:

Theorem 2.1. The Schwartz space $\mathcal{S}$ consists of all infinitely differentiable functions $\varphi$ satisfying the condition (2.2).

The proof of Theorem 2.1 in [3] is based on the combination of induction and the method of proof of the Sobolev embedding theorem. We give here a very elementary proof of Theorem 2.1 based on the elementary inequality of Landau concerning the estimates of derivatives as in Duistermaat [8, p.40].

Proof of Theorem 2.1. It suffices to show that the condition (2.2) implies (2.1) since its converse is trivial. Assume that an infinitely differentiable function $\varphi$ satisfies (2.2). Put $x=\left(x_{1}, x^{\prime}\right)$, and also assume that $x_{1}>0$ and $h>0$ for a moment. Then the Taylor expansion of $\varphi$ at $x$ with respect to the $x_{1}$ variable gives

$$
\varphi\left(x_{1}+h, x^{\prime}\right)=\varphi\left(x_{1}, x^{\prime}\right)+h \partial_{1} \varphi\left(x_{1}, x^{\prime}\right)+\frac{h^{2}}{2} \partial_{1}^{2} \varphi\left(\xi, x^{\prime}\right)
$$


for some $\xi$ with $x_{1}<\xi<x_{1}+h$. It follows from the first condition of (2.2) that for any positive integer $N$ there exists a constant $C=C(N)$ such that

$$
|\varphi(x)| \leq C\left(1+|x|^{2}\right)^{-N}, \quad\left|\varphi\left(x_{1}+h, x^{\prime}\right)\right| \leq C\left(1+|x|^{2}\right)^{-N}, \quad x_{1}>0,
$$

for all $h>0$. Put $M=\sup \left|\partial_{1}^{2} \varphi(x)\right|$, which is finite by the second condition of (2.2). Since it follows from $(2.3)$ that

$$
\partial_{1} \varphi\left(x_{1}, x^{\prime}\right) \leq \frac{1}{h}\left(\left|\varphi\left(x_{1}+h, x^{\prime}\right)\right|+\left|\varphi\left(x_{1}, x^{\prime}\right)\right|\right)+\frac{h}{2}\left|\partial_{1}^{2} \varphi\left(\xi, x^{\prime}\right)\right|
$$

for all $h>0$, we obtain

$$
\left|\partial_{1} \varphi(x)\right|^{2} \leq 2 M C\left(1+|x|^{2}\right)^{-N}, \quad x_{1}>0 .
$$

Therefore, for any $\alpha$ we also obtain

$$
\left|x^{\alpha} \partial_{1} \varphi(x)\right|^{2} \leq 2 M C|x|^{2|\alpha|}\left(1+|x|^{2}\right)^{-N}, \quad x_{1}>0,
$$

if we choose $N>|\alpha|$. Thus it follows that $\sup _{x_{1}>0}\left|x^{\alpha} \partial_{1} \varphi(x)\right|<\infty$. Now if we put $\psi(x)=\varphi\left(-x_{1}, x^{\prime}\right)$, then $\psi$ also satisfies (2.2), which implies that

$$
\sup _{x_{1}>0}\left|x^{\alpha} \partial_{1} \psi(x)\right|=\sup _{x_{1}<0}\left|x^{\alpha} \partial_{1} \varphi(x)\right|<\infty .
$$

Therefore, it is clear that $\sup \left|x^{\alpha} \partial_{1} \varphi(x)\right|<\infty$ for any $\alpha$. Similarly, we have $\sup \left|x^{\alpha} \partial_{i} \varphi(x)\right|<\infty$ for $i=1, \cdots, n$, which implies that the class characterized by the condition (2.2) is stable under the differentiation. Therefore, it follows that $\sup \left|x^{\alpha} \partial^{\beta} \varphi(x)\right|<\infty$ for any $\beta$, which completes the proof.

We now state the following corollary as in [3], which is more symmetric relative to the Fourier transform and refer to [3] for an easy proof.

Corollary 2.2. The Schwartz space $\mathcal{S}$ is characterized by the two inequalities

$$
\sup _{x}\left|x^{\alpha} \varphi(x)\right|<\infty, \quad \sup _{\xi}\left|\xi^{\beta} \hat{\varphi}(\xi)\right|<\infty
$$

for all $\alpha$ and $\beta$.

Remark 2.3. Combining Corollary 2.2 and the above result II in Section 1 on the Sato space $\mathcal{F}$ in [4] we can easily compare the spaces $\mathcal{S}$ and $\mathcal{F}$ which are both invariant under the Fourier transformation. In the theory of Schwartz distributions, thinking of duality $\langle u, \varphi\rangle$ we can take the Fourier transform of tempered, i.e., polynomially increasing, functions or distributions. On the other hand, in the Sato theory of Fourier hyperfunctions we can take the Fourier transform of infraexponentially tempered functions or hyperfunctions. Here a function $f$ is said to be infra-exponentially tempered or slowly increasing if for every $\epsilon>0$ there is a constant $C$ such that $|f(x)|<C \exp (\epsilon|x|)$ for every $x$. This clarifies the theory of T. Carleman [2] on Fourier integrals.

Also, we can easily apply the above method to the following spaces $H\left(\mathbb{R}^{n}\right), S_{1}$ and $\mathcal{S}_{E}$, introduced by Hasumi, Gelfand-Shilov and Chung-Kim in [10], [9], [6] respectively as follows:

$$
\begin{aligned}
H\left(\mathbb{R}^{n}\right) & =\left\{\varphi \in C^{\infty}\left|{ }^{\forall} \alpha^{\forall} k \sup _{x}\right| \partial^{\alpha} \varphi(x)|\exp k| x \mid<\infty\right\}, \\
S_{1} & =\left\{\varphi \in C^{\infty}\left|{ }^{\exists} k^{\forall} \alpha \sup _{x}\right| \partial^{\alpha} \varphi(x)|\exp k| x \mid<\infty\right\}, \\
\mathcal{S}_{E} & =\left\{\varphi \in C^{\infty}\left|{ }^{\forall} \alpha{ }^{\exists} k \sup _{x}\right| \partial^{\alpha} \varphi(x)|\exp k| x \mid<\infty\right\} .
\end{aligned}
$$


In other words, we can give a better definition for the above spaces as follows:

$$
\begin{aligned}
H\left(\mathbb{R}^{n}\right) & =\left\{\varphi \in C^{\infty}\left|{ }^{\forall} \alpha^{\forall} k \sup _{x}\right| \varphi(x)|\exp k| x\left|<\infty, \sup _{x}\right| \partial^{\alpha} \varphi(x) \mid<\infty\right\}, \\
S_{1} & =\left\{\varphi \in C^{\infty}\left|{ }^{\exists} k^{\forall} \alpha \sup _{x}\right| \varphi(x)|\exp k| x\left|<\infty, \sup _{x}\right| \partial^{\alpha} \varphi(x) \mid<\infty\right\}, \\
\mathcal{S}_{E} & =\left\{\varphi \in C^{\infty}\left|{ }^{\forall} \alpha^{\exists} k \sup _{x}\right| \varphi(x)|\exp k| x\left|<\infty, \sup _{x}\right| \partial^{\alpha} \varphi(x) \mid<\infty\right\} .
\end{aligned}
$$

\section{Better Characterization of the Beurling-BjörCK SPACE}

G. Björck introduced the space Beurling-Björck space $\mathcal{S}_{\omega}$ of test functions for tempered ultradistributions to extend the space $\mathcal{S}^{\prime}$ of tempered distributions in [1] as follows.

Definition 3.1. By $\mathcal{S}_{\omega}$ or $\mathcal{S}_{\omega}\left(\mathbb{R}^{n}\right)$ we denote the set of all $\phi \in C^{\infty}\left(\mathbb{R}^{n}\right) \cap L^{1}\left(\mathbb{R}^{n}\right)$ such that

$$
\begin{aligned}
& p_{\alpha, \lambda}(\varphi)=\sup _{x \in \mathbb{R}^{n}} e^{\lambda \omega(x)}\left|\partial^{\alpha} \varphi(x)\right|<\infty, \\
& \pi_{\alpha, \lambda}(\varphi)=\sup _{\xi \in \mathbb{R}^{n}} e^{\lambda \omega(\xi)}\left|\partial^{\alpha} \hat{\varphi}(\xi)\right|<\infty
\end{aligned}
$$

for each multi-index $\alpha$ and each nonnegative number $\lambda$, where the continuous weight function $\omega$ on $\mathbb{R}^{n}$ satisfies the following:

$(\gamma) \omega(\xi) \geq a+b \log (1+|\xi|), \quad \xi \in \mathbb{R}^{n}, \quad$ for some real $a$ and positive $b$,

$(\delta) \omega(\xi)=\Omega(|\xi|)$ with $\Omega$ concave on $[0, \infty)$.

Remark 3.2. In [1] G. Björck imposed the subadditivity $(\alpha)$ and non quasianalyticity $(\beta)$ conditions in addition, but they are not necessary here.

We now show that the assumption for the decay of derivatives in Definition 3.1 is redundant:

Theorem 3.3. Let $\omega$ be a function satisfying the conditions $(\gamma)$ and $(\delta)$ and let $\varphi$ and $\hat{\varphi}$ be $C^{\infty}$ functions. Then the following are equivalent.

(i) $\sup _{x \in \mathbb{R}^{n}} e^{\lambda \omega(x)}\left|\partial^{\alpha} \varphi(x)\right|<\infty, \quad \sup _{\xi \in \mathbb{R}^{n}} e^{\lambda \omega(\xi)}\left|\partial^{\alpha} \hat{\varphi}(\xi)\right|<\infty$,

(ii) $\sup _{x \in \mathbb{R}^{n}} e^{\lambda \omega(x)}|\varphi(x)|<\infty, \quad \sup _{\xi \in \mathbb{R}^{n}} e^{\lambda \omega(\xi)}|\hat{\varphi}(\xi)|<\infty$

for all $\lambda>0$ and $\alpha$.

Proof. It suffices to show that (ii) implies (i). Now assume that $\varphi$ satisfies (ii). It follows from (ii) and Corollary 2.2 that $\varphi$ belongs to the Schwartz space $\mathcal{S}$. Also, since the conditions $(\gamma)$ and $(\delta)$ imply that $\omega(\xi)$ is radially increasing, i.e., $\omega(\xi)>\omega(\eta)$ for $|\xi|>|\eta|$, there exists a positive constant $C^{\prime}$ such that for all $h>0$ and $x$

$$
|\varphi(x)|<C^{\prime} e^{-\lambda \omega(x)}, \quad\left|\varphi\left(x_{1}+h, x^{\prime}\right)\right| \leq C^{\prime} e^{-\lambda \omega(x)} .
$$

The Fourier inversion formula gives

$$
\left|\partial_{1}^{2} \varphi(x)\right| \leq \int\left|\xi_{1}\right|^{2} e^{-\lambda \omega(\xi)} e^{\lambda \omega(\xi)}|\hat{\varphi}(\xi)| d \xi \leq C \int\left|\xi_{1}\right|^{2} e^{-\lambda \omega(\xi)} d \xi
$$

for all $\lambda$. Note that $e^{-\lambda \omega(\xi)} \leq e^{-\lambda \log \left(e^{a}(1+|\xi|)^{b}\right)}=e^{-\lambda a}(1+|\xi|)^{-\lambda b}$. So, if we take $\lambda b>n+2$ the last term of (3.2) is integrable. Now let $M$ be the upper bound of $\left|\partial_{1}^{2} \varphi(x)\right|$. Then it follows from (2.4) and (3.1) that for all $h>0$

$$
\left|\partial_{1} \varphi(x)\right| \leq \frac{2}{h} C^{\prime} e^{-\lambda \omega(x)}+\frac{h}{2} M .
$$


Therefore, we can easily see that

$$
\left|\partial_{1} \varphi(x)\right| \leq 2 \sqrt{C^{\prime} e^{-\lambda \omega(x)} M} \leq C^{\prime \prime} e^{-\lambda \omega(x) / 2},
$$

which implies that

$$
\sup _{x_{1}>0} e^{\lambda \omega(x) / 2}\left|\partial_{1} \varphi(x)\right|<\infty .
$$

Applying the same method as in the proof of Theorem 2.1 we obtain

$$
\sup e^{\lambda \omega(x) / 2}\left|\partial_{1} \varphi(x)\right|<\infty,
$$

and the same estimate (3.3) for $\left|\partial_{i} \varphi(x)\right|, i=2, \ldots, n$, and finally the first inequality of (i).

For the second inequality of (i) we have to estimate the Fourier transform $\hat{\varphi}$. For this note that $\left|\partial_{1}^{2} \hat{\varphi}(\xi)\right| \leq \int\left|x_{1}\right|^{2}|\varphi(x) d x|<\infty$. Applying a similar method as above we obtain that $\left|\partial_{i} \hat{\varphi}(\xi)\right| \leq C e^{-\lambda \omega(\xi) / 2}$ for $i=1, \ldots, n$, and finally the second inequality of (i), which completes the proof.

In order to compare the Beurling-Björck spaces with the Silva space $\mathcal{G}$ of test functions for the Fourier ultrahyperfunctions of Sebastião e Silva and MorimotoPark as in [7] we characterize the Silva space $\mathcal{G}$ as follows.

Theorem 3.4. The Silva space $\mathcal{G}$ consists of all locally integrable functions such that for any $k>0$

$$
\sup _{x}|\varphi(x)| \exp k|x|<\infty, \quad \sup _{\xi}|\hat{\varphi}(\xi)| \exp k|\xi|<\infty .
$$

In fact, applying a similar method to the Silva space $\mathcal{G}$ in [7] as in [4] for the Sato space $\mathcal{F}$ we can prove the above theorem without much difficulty.

By Theorem 3.4 we can easily see that the Silva space $\mathcal{G}$ is a special case of the Beurling-Björck space when $\omega(x)=|x|$.

\section{REFERENCES}

1. G. Björck, Linear partial differential operators and generalized distributions, Ark. Mat. 6 (1965), 351-407. MR 34:3054

2. T. Carleman, L'intégral de Fourier et questions qui s'y rattachent, Publ. Sci. Inst. MittagLeffler, Uppsala, 1944.

3. J. Chung, S. Y. Chung and D. Kim, Une caractérisation de l'espace de Schwartz, C. R. Acad. Sci. Paris Sér. I Math. 316 (1993), 23-25. MR 93m:46040

4. - A characterization for Fourier hyperfunctions, Publ. RIMS, Kyoto Univ. 30 (1994), 203-208. MR 94m:46066

5. _ A characterization of the Gelfand-Shilov spaces via Fourier transform, Proc. Amer. Math. Soc. 124 (1996), 2101-2108. MR 96i:46043

6. S.-Y. Chung and D. Kim, Distributions with exponential growth and Bochner-Schwartz theorem for Fourier hyperfunctions, Publ. RIMS, Kyoto Univ. 31 (1995), 829-845. MR 96m:46074

7. S.-Y. Chung, D. Kim and S. K. Kim, Structure of the extended Fourier hyperfunctions, Japan. J. Math. 19 (1993), 217-226. MR 94m:46075

8. J. Duistermaat, Fourier integral operators, Courant Institute, New York, 1973. MR 56:9600

9. I.M. Gelfand and G.E. Shilov, Generalized functions II, Academic Press, New York, 1968. MR 55:8786b

10. M. Hasumi, Note on the n-dimensional tempered ultra-distributions, Tôhoku Math. J. 13 (1961), 94-104. MR 24:A1607 
11. H.-J. Schmeisser and H. Triebel, Topics in Fourier analysis and function spaces, Wiley, New York, 1987. MR 88k:42015b

12. L. Schwartz, Théorie des distributions, Hermann, Paris, 1966. MR 35:730

Department of Mathematics, Sogang University, Seoul 121-742, Korea

E-mail address: sychung@ccs.sogang.ac.kr

Department of Mathematics, Seoul National University, Seoul 151-742, Korea

E-mail address: dhkim@math.snu.ac.kr

Department of Mathematics, Daejin University, Pochun 487-800, Korea

E-mail address: hyper@math.snu.ac.kr 